\title{
Giving Blood After the Schoolyard Shootings
}

\section{A transparent man}

on the blood bank wall his body crowded

with the branching and sprouting of the circulatory system

what happens

inside the heart contracting its red

arterial blood swelling the ventricle the blue veins draining

the vena cava

emptying the limbs she swabs my arm

inserts a needle and hangs the plastic bladder out of sight

I squeeze and release

a child's red ball the blood jets

blackening the tube the clinic bustling like Intensive Care

red flecks

speckling the tile a red jewel

in the nurse's ear the sunset coloring the flooded pavement

this letting

these ruby pools in the parking lot

a woman in the next chair describing an article about dreams

she hadn't known

how the eye must keep moving even

asleep it roams its socket to keep the fluids moving it might

dry up otherwise

a dream is just something for it

to seem to see they're meaningless dreams it's just a theory. 